\title{
Effects of fullerene nanowhiskers on cytotoxicity and gene expression
}

\author{
Junko Okuda-Shimazaki ${ }^{1}$, Shinichi Nudejima ${ }^{2}$, Saiko Takaku ${ }^{1}$, Koki Kanehira $^{3}$, Shuji Sonezaki ${ }^{3}$ \\ Akiyohshi Taniguchi $^{{ }^{*}}$

\footnotetext{
${ }^{1}$ Advanced Medical Materials Group, Biomaterials Center, National Institute for Materials Science, Namiki, Tsukuba, Ibaraki, Japan;

${ }^{2}$ Advanced Nanomaterials Laboratory, National Institute for Materials Science, Namiki, Tsukuba, Ibaraki, Japan;

${ }^{3}$ TOTO Ltd. Research Institute, Nakashima, Kokurakita, Kitakyushu, Japan; *Corresponding Author: taniguchi.akiyoshi@nims.go.jp
}

Received 23 June 2010; revised 10 July 2010; accepted 14 July 2010

\begin{abstract}
With recent developments in nanoscience and nanotechnology, carbon-based nanomaterials, such as carbon nanotubes or Fullerene nanowhiskers (FNWs), are attracting a great deal of interest. However, nanomaterials have novel properties that may cause safety problems in biological systems. Although the toxic effects of multiwall carbon nanotubes (MWCNTs) in vitro and in vivo have been well described, the effect of FNWs on cells remains unclear. In the present study, we analyzed the gene expression and cytotoxicity of FNWs-treated cells. The results of cell viability and gene expression analysis indicate that FNWs has a relatively smaller ability to induce cellular gene expression as compared with MWCNTs or titania nanoparticles. Our results suggest that FNWs have weak cytotoxic effects as compared to the effects of MWCNTs and titania nanoparticles.
\end{abstract}

Keywords: Nanomaterials; Cytotoxicity; Gene Expression; Fullerene Nanowhisker

\section{INTRODUCTION}

Nanomaterials are currently being investigated and have potential applications in various fields. With recent developments in nanoscience and nanotechnology, carbon-based nanomaterials, such as carbon nanotubes (CNTs) and Fullerene nanowhiskers (FNWs), are attracting a great deal of interest. Because of their size, chemical composition, surface structure, shape, and aggregation properties, nanomaterials are expected to have novel physicochemical properties. The small size of nanomaterials allows them to penetrate into the body [1] These novel properties raise safety concerns for the use of nanomaterials in biological systems. Recent studies suggest that nanomaterials affect biological behavior and have the potential to be toxic [2-6]. The toxic effects of multiwall carbon nanotubes (MWCNTs) in vitro and in vivo have been well described [5,7-11]. FNWs also have the potential to be used as carbonaceous engineered nanomaterials in the future. However, the effect of FNWs on cells remains unclear.

In the present study, to understand the biological activity of FNWs, we analyzed the gene expression and cytotoxicity of FNWs-treated cells. Our results suggest that FNWs have a very weak effect on cytotoxic activity and the induction of gene expression as compared to the effects of MWCNTs and titania nanoparticles.

\section{MATERIALS AND METHODS}

\subsection{Nanomaterials}

FNWs, which were formed by the liquid-liquid interfacial precipitation method [12-14], were a kind gift from Dr K. Miyazawa (National Institute for Materials Science). MWCNTs (MITSUI MWNT-7; Lot. 06122002) were a kind gift from Dr. S. Tsuruoka (Mitsui \& Co.,Ltd.). Small aggregated titania nanoparticles were prepared by the centrifuge method [6].

\subsection{Cell Cultures}

A human acute monocytic leukemia cell line, THP-1 [14,15], was cultured in RPMI 1640 medium supplemented with $10 \%$ fetal bovine serum, $100 \mathrm{U} / \mathrm{mL}$ penicillin, and $100 \mu \mathrm{g} / \mathrm{mL}$ streptomycin under $5 \% \mathrm{CO}_{2}$ with $100 \%$ humidity at $37^{\circ} \mathrm{C}$. For the exposure experiments, THP-1 cells were treated with $200 \mathrm{nM}$ phorbol 12- myristate 13-acetate (PMA) for $48 \mathrm{~h}$. To expose the cells, PMA-treated THP-1 cells that had been seeded $24 \mathrm{~h}$ prior were exposed to titania particles for $24 \mathrm{~h}$. 


\subsection{Microscopic Observation}

Cells exposed to nanomaterials were fixed with paraformaldehyde and stained with Hoechst 33342 (nucleus marker) and rhodamine-phalloidin (F-actin marker). Microscopic images of fixed cells were obtained by laser scanning microscopy.

\subsection{Cell Viability Test}

Cell viability was measured by using a CellTiter-Glo Luminescent Cell Viability Assay kit (Promega). THP-1 cells $\left(5.0 \times 10^{4}\right.$ per well $)$ were seeded in a 96 -well cell culture plate. Titania particle suspensions were prepared at final concentrations from $0.00001 \% \mathrm{w} / \mathrm{v}(0.1 \mu \mathrm{g} / \mathrm{mL})$ to $0.001 \% \mathrm{w} / \mathrm{v}(10 \mu \mathrm{g} / \mathrm{mL})$. Each concentration of titania particle suspension was added to the cell culture medium at a 1/100 volume, and the cells were cultured for $24 \mathrm{~h}$ prior to adding a reagent mixture containing cell lysis solution, luciferase, and luciferase substrate to the wells. The luminescence of the luciferase reaction, which depends on the cytoplasmic ATP concentration, was then analyzed.

\subsection{Gene Expression Analysis}

For mRNA expression analysis, THP-1 cells $(1.4 \times$ $104 \mathrm{~cm}^{2}$ ) were seeded in cell culture dishes. Nanomaterials were prepared at a final concentration of $100 \mu \mathrm{g} / \mathrm{mL}$. Following $24 \mathrm{~h}$ of exposure to nanomaterials, cells were detached by mechanical dissociation and utilized for gene expression analysis.

The expression levels of marker genes were determined by quantitative real-time RT-PCR as described previously [6]. Total cellular RNA was extracted from titania-exposed cells by using an RNeasy Kit (Qiagen). Extracted RNA was treated with DNaseI. Total cellular RNA $(2 \mu \mathrm{g})$ was reversibly transcribed with a random hexamer primer by using the SuperScript III First-Strand Synthesis System for RT-PCR (Invitrogen). The cDNA $(2 \mu \mathrm{L})$ was mixed with $10 \mu \mathrm{L}$ of $2 \mathrm{x}$ Master Mix from the qPCR Mastermix Plus for SYBR Green I kit (Takara, Japan) and with 10 pmol of each specific primer. The PCR was performed as previously described [6]. Expression levels of marker genes were normalized to that of a housekeeping gene, glyceraldehyde-3-phosphate dehydrogenase (GAPDH), which was used as an endogenous control in the same reaction as the gene of interest. The primers for qPCR were as follows: for GAPDH, forward 5'-CCCCCACCACACTGAATCTC- 3' and reverse 5'-GCCCCTCCCCTCTTCAAG-3'; and for Interleukin 6 (IL6), forward 5'-TGAGTACAAAAGTCCT GA-3' and reverse 5'-TCTGTGCCTGCAGCTTCGT-3'. The results from at least three independent tests were evaluated by using Dunnett's multiple comparison test.

\subsection{DNA Microarray Analysis}

One microgram of total RNAs was amplified and labeled by using an Amino Allyl MessageAmp aRNA kit (Ambion, Austin, TX). The DNA microarray, AceGene- 1 Chip Version-Mouse (Hitachi Software Engineering, Tokyo, Japan) with about 30,000 oligo-sense-nucleotides including 22,917 unique genes on the basis of GeneID, was covered with a gap cover glass (Takara), the solution was injected from the edge of the cover glass, and the microarray was placed in a hybridization cassette (TaKaRa Bio). The DNA microarray was dried by centrifugation and scanned by using GenePix4000 (Axon Instruments, Silicon Valley, CA) to detect the array image.

\section{RESULTS}

\subsection{Microscopic Images of FNWs-Exposed Cells}

Exposure tests were performed with a human monocytic cell line, THP-1. THP-1 cells were differentiated, before FNWs exposure, by the addition of PMA for phagocytosis. Microscopic images of FNWs-exposed cells suggested that the FNWs were taken up by THP-1 cells and localized in the cytoplasmic space (Figure 1).

\subsection{Viability of FNWs-Exposed Cells}

We measured the viability of cells exposed to FNWs or MWCNTs (as a control) based on the quantification of the cytoplasmic ATP concentration, which signals the presence of metabolically active cells. Ninety percent of FNWs-and MWCNTs-exposed THP-1 cells were viable
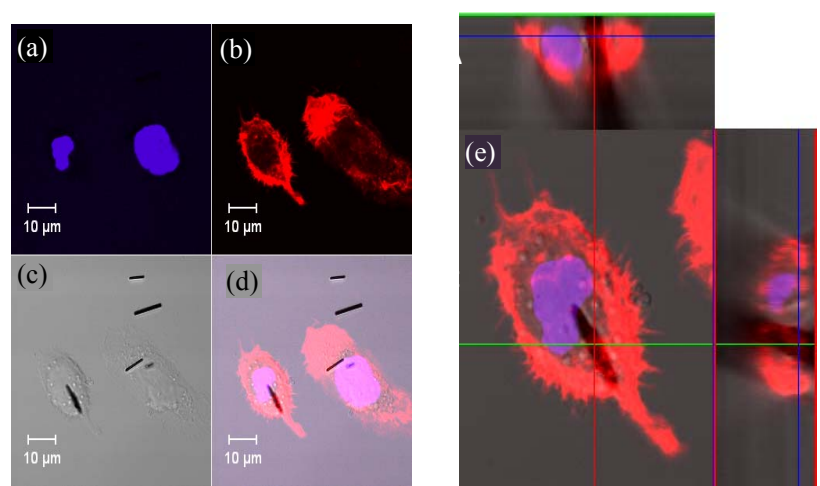

Figure 1. Microscopic images of FNWs-exposed THP-1 cells. FNWs-exposed cells were fixed with PFA and stained with Hoechst 33342 (a) and rhodamine-phalloidin (b) FNWs were observed in differential interference images (c) Images represent the nucleus (a), F-actin (b), differential interference images (c), merged images (d), and Z-section images of FNWs- exposed cells (e). 


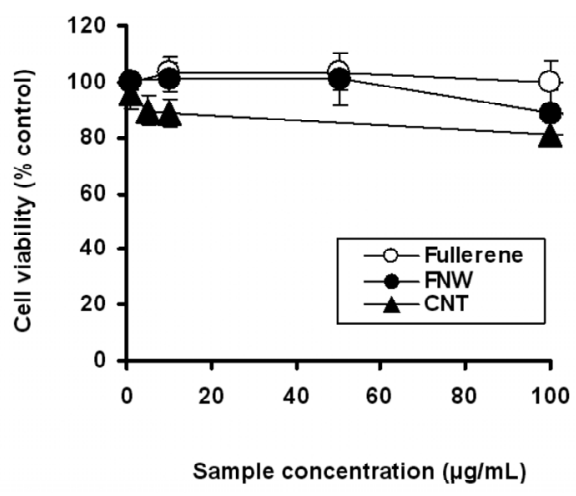

Figure 2. Cell viability test based on cytoplasmic ATP concentrations. Cell viability was measured by using a CellTiter-Glo Luminescent Cell Viability Assay kit (Promega). THP-1 cells were exposed to Fullerene as a control (closed circle) or FNWs (open circle) or MWCNTs (closed triangle).

at high concentrations of FNWSS and MWCNTs (Figure 2). There were no significant differences between the viability of FNWs-exposed cells and MWCNTsexposed cells. Thus, FNWs had very weak cytotoxic activity at these concentrations.

\subsection{IL-6 mRNA Expression in FNWs-exposed Cells}

We next investigated the mRNA expression of the IL-6 gene in FNWs-exposed cells. We previously showed that the IL-6 gene is a good marker for detecting the cytotoxicity of nanomaterials [6]. The expression of IL6 mRNA was induced in THP-1 cells exposed to MWCNTs (Figure 3). There was no apparent change in expression in FNWs-exposed cells. These results indicate that FNWs has a relatively smaller ability to induce cellular gene expression as compared to MWCNTs.

\subsection{DNA Microarray Analysis}

We used DNA microarray analysis to compare the gene expression profiles of THP-1 cells exposed to FNWs and titania nanoparticles (Table 1). A total of 576 genes were induced by more than 4 -fold after titania

Table 1. Number of up-or down-regulated genes by nanomaterials using DNA microarray analysis.

\begin{tabular}{ccc}
\hline Fold induction & Titania vs control & ENW vs control \\
\hline $4<$ & 8 & 1 \\
$2<<4$ & 568 & 2 \\
$1.5<<2$ & 729 & 22 \\
$-1.5<<1.5$ & 18087 & 20869 \\
$-2>>-4$ & 78 & 254 \\
$-2>>-4$ & 42 & 17 \\
$-4>$ & 2 & 0 \\
\hline
\end{tabular}

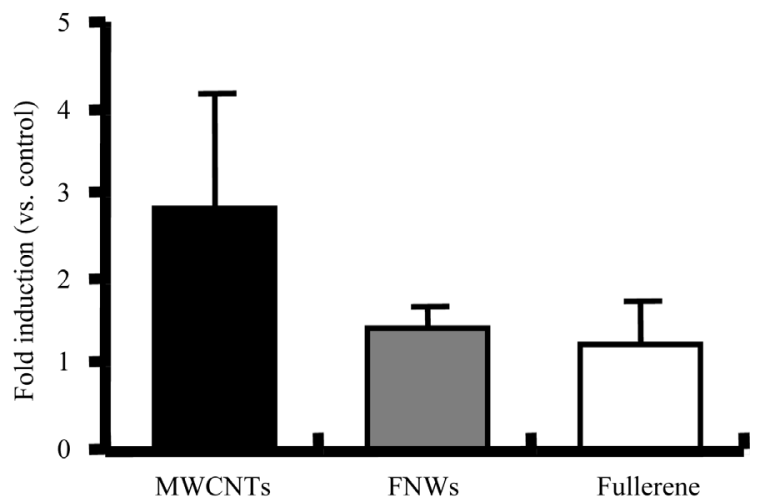

Figure 3. IL-6 mRNA expression of nanomaterials-exposed THP-1 cells. The expression levels of IL-6 gene was determined by quantitative real-time RT-PCR method. PMA-activated THP-1 cells were exposed to $100 \mu \mathrm{g} / \mathrm{mL}$ Fullerene (solid bar) or $100 \mu \mathrm{g} / \mathrm{mL}$ FNWs (gray bar) or $100 \mu \mathrm{g} / \mathrm{mL}$ MWCNTs for $24 \mathrm{~h}$. mRNA expression was standardized by internal GAPDH (glyceraldehyde-3-phosphate dehydrogenase) expression, and the relative expression level versus control (isopropyl alcohol was added instead of nanomaterials) is shown.

nanoparticle exposure. On the other hand, only three genes were induced by more than 4 -fold after FNWs exposure. These results indicate that FNWs has a relatively smaller ability to induce cellular gene expression as compared with titania nanoparticles.

\section{DISCUSSION}

In the present study, we analyzed the biological activity of FNWs. The physicochemical properties of FNWs, such as size and chemical composition, were very similar to those of MWCNT. However, our results suggest that FNWs had a very weak effect on cytotoxic activity and the induction of gene expression as compared to MWCNTs.

We previously studied the phagocytosis of FNWs by PMA-treated THP1 cells $[14,16]$. Our results suggested that PMA-treated THP1 cells might be able to decompose FNWs into fullerene molecules. The weak effects of FNWs on cytotoxic activity and the induction of gene expression might be due to the biodegradable property of FNWs in cells.

\section{ACKNOWLEDGMENT}

We thank the Bio-Organic Materials Facility at the Nanotechnology Innovation Center for technical assistance. Thanks Dr. K. Miyazawa (National Institute for Materials Science) and Dr. S. Tsuruoka (Mitsui \& Co.,Ltd.) for kind gift FNWs and MWCNTs.

\section{REFERENCES}

[1] Nel, A., Xia, T., Mädler, L., Li, N. (2006) Toxic potential 
of materials at the nanolevel. Science, 311, 622- 627.

[2] Service, R.F. (2004) Nanotoxicology. Nanotechnology grows up. Science, 304, 1732-1734.

[3] Oberdörster, G., Oberdörster, E., Oberdörster, J. (2005) Nanotoxicology: An emerging discipline evolving from studies of ultrafine particles. Environ. Health Perspect. 113, 823-839.

[4] Poland, C.A., Duffin, R., Kinloch, I., et al. (2008) Carbon nanotubes introduced into the abdominal cavity of mice show asbestos-like pathogenicity in a pilot study. Nat. Nanotechnol., 3, 423-428.

[5] Takagi, A., Hirose, A., Nishimura, T., et al. (2008) Induction of mesothelioma in $\mathrm{p} 53+/-$ mouse by intraperitoneal application of multi-wall carbon nanotube. Journal of Toxicological Sciences, 33, 105-116.

[6] Okuda-Shimazaki, J., Takaku, S., Kanehira, K., et al. (2010) Aggregate particle size effects of titanium dioxide nanoparticles on the gene expression. International Journal of Molecular Sciences, 11, 2383-2392.

[7] Patlolla, A., Patlolla, B., Tchounwou, P. (2010) Evaluation of cell viability, DNA damage, and cell death in normal human dermal fibroblast cells induced by functionalized multiwalled carbon nanotube. Molecular and Cellular Biochemistry, 338, 225-232.

[8] Jos, A., Pichardo, S., Puerto, M., et al. (2009) Cytotoxicity of carboxylic acid functionalized single wall carbon nanotubes on the human intestinal cell line Caco-2. Toxicol In Vitro. 23, 1491-1496.

[9] Lin, C., Fugetsu, B., Su, Y., Watari, F. (2009) Studies on toxicity of multi-walled carbon nanotubes on Arabidopsis T87 suspension cells. Journal of Hazard Mater. 170, 578-583.

[10] Tong, H., McGee, J.K., Saxena, R.K., et al. (2009) Influence of acid functionalization on the cardiopulmonary toxicity of carbon nanotubes and carbon black particles in mice. Toxicology and Applied Pharmacology, 239, 224-232.

[11] Belyanskaya, L., Weigel, S., Hirsch, C., et al. (2009) Effects of carbon nanotubes on primary neurons and glial cells. Neurotoxicology. 30, 702-711.

[12] Miyazawa, K., Kuwasaki, Y., Obayashi, A., Kuwabara, M. (2002) C60 nanowhiskers formed by the liquid-liquid interfacial precipitation method. Journal of Materials Research, 17, 83-88.

[13] Miyazawa, K., Cha, S., Ringor, C., et al. (2008) Synthesis of fullerene nanotubes and microtubes for materials storage, delivery and recovery. Nano, 3, 335-339.

[14] Nudejima S., Miyazawa K., Okuda J., et al. (2009) Observation of phagocytosis of fullerene nanowhiskers by PMA-treated THP-1 cells. Journal of Physics, 159, 1-6.

[15] Okuda-Shimazaki, J., Yamamoto, A., Kuroda, D., et al. (2007) The effect of metal materials on heat shock protein 70B' gene expression. Open Biotechnology Journal, 1, 14-17.

[16] Nudejima S, Miyazawa K, Okuda-Shimazaki J et al. (2010) Biodegradation of C60 fullerene nanowhiskers by macrophage-like cells. Advances In Biomedical Research, pp.89-94 